\title{
Experimental and Numerical Analysis of High-Resolution Injection Technique for Capillary Electrophoresis Microchip
}

Chin-Lung Chang ${ }^{1}$, Jik-Chang Leong ${ }^{1}$, Ting-Fu Hong ${ }^{2}$, Yao-Nan Wang ${ }^{1}{ }^{*}$ and Lung-Ming Fu ${ }^{2, *}$

1 Department of Vehicle Engineering, National Pingtung University of Science and Technology, Pingtung 912, Taiwan; E-Mails: clchang@mail.npust.edu.tw (C.-L.C.); jcleong@mail.npust.edu.tw (J.-C.L.)

2 Department of Materials Engineering, National Pingtung University of Science and Technology, Pingtung 912, Taiwan; E-Mail: tfhong@ mail.npust.edu.tw

* Authors to whom correspondence should be addressed; E-Mails: ynwang @ npust.edu.tw (Y.-N.W.); loudyfu@mail.npust.edu.tw (L.-M.F.); Tel.: +886-8-77032020 ext. 7553; Fax: +886-8-7740552.

Received: 30 March 2011; in revised form: 11 May 2011 / Accepted: 25 May 2011 /

Published: 3 June 2011

\begin{abstract}
This study presents an experimental and numerical investigation on the use of high-resolution injection techniques to deliver sample plugs within a capillary electrophoresis (CE) microchip. The CE microfluidic device was integrated into a U-shaped injection system and an expansion chamber located at the inlet of the separation channel, which can miniize the sample leakage effect and deliver a high-quality sample plug into the separation channel so that the detection performance of the device is enhanced. The proposed $45^{\circ} \mathrm{U}$-shaped injection system was investigated using a sample of Rhodamine B dye. Meanwhile, the analysis of the current CE microfluidic chip was studied by considering the separation of Hae III digested $\varphi x-174$ DNA samples. The experimental and numerical results indicate that the included $45^{\circ} \mathrm{U}$-shaped injector completely eliminates the sample leakage and an expansion separation channel with an expansion ratio of 2.5 delivers a sample plug with a perfect detection shape and highest concentration intensity, hence enabling an optimal injection and separation performance.
\end{abstract}

Keywords: capillary electrophoresis microchip; injection system; expansion chamber 


\section{Introduction}

In recent years, integrated microfluidic devices, also known as "Lab-on-a-Chip", have been utilized for the analysis of chemical or biological assays in biochemistry, biophysics, medicine and life sciences [1-10]. Microfluidic chips offer researchers many potential benefits, including a high sample throughput, integrated sample processing, minimized sample and reagent volume, improved performance and reliability, and integration of the chip and detection circuitry. Now, many functional microfluidic devices have been developed to perform a variety of tasks, including sample pre-treatment and injection, species mixing, polymerase chain reaction, and cell/particle separation and counting [11-21].

The injector and separation channel are the key elements of a CE microfluidic device, which is a cross-shaped microchannel that uses electrokinetic flow to load samples into the intersection of one channel and dispenses a minute quantity of sample into the separation channel. The precise control of the sample injection and separation processes in microfluidic dispensers is very important to the analysis. One of the most common sample injection methods in CE microfluidic devices is the cross-shaped injection technique. The floating sample injection method causes the sample leakage to increase the signal baseline as the number of injection runs increases, thus reducing the separation efficiency [22-27]. Fu et al. [22] identified severe sample leakage in the floating sample injection method when high voltage gradients were established. The sample leakage effect was found to increase the signal baseline as the number of injection runs increased resulting in the reduction of the separation efficiency. Consequently, the authors developed a low-leakage injection technique designed specifically to improve the detection performance of microfluidic devices. Zhuang et al. [27] developed a novel electrokinetic double-focusing injection technique for a capillary electrophoresis microchip, which involved four accessory arm channels in which symmetrical focusing potentials were loaded to form a unique parallel electric field distribution at the intersection of the injection channel and separation channel. The parallel electric field effectuates virtual walls to confine the spreading of the sample plug at the intersection and prevents sample leakage into the separation channel.

Electrokinetic focusing may be used to create a variable sample plug volume injector that produces consistent sample plug volumes. The improved sample plug distribution in the separation channel was reported to provide an enhanced separation performance [28,29]. Luo et al. [30] presented a double-cross hydrostatic pressure sample injection for a capillary electrophoresis microchip. The injection method uses hydrostatic pressure generated by emptying the sample waste reservoir for sample loading and electrokinetic force for dispensing. One cross, created by the sample and separation channels, is used for the formation of a sample plug. Another cross, formed by the sample and controlling channels, is used for plug control. This injection method enables linear adjustment of the sample plug volume and has the ability to reduce the number of electrodes to a minimum for either single- or multi-channel electrophoresis.

Some studies related to the improved sample injection and separation technique in microchip electrophoresis have been performed. For example, Wu and Yang [31] presented a T-form electrokinetic injection system for the discrete time-based loading and dispensing of samples of variable-volume in a microfluidic chip. The push-pull effect, which is produced during the loading 
and dispensing processes, can shape a compact sample plug and hence improve the detection resolution of the microfluidic device. Wenclawiak and Püschl [32] showed a mini-review of the sample injection for capillary electrophoresis on a micro fabricated device with on-chip CE injection. They introduced different injection designs (including T-type, double T-type, and cross) and different injection modes (such as pinched injection, gated injection, optically gated injection, pressure/pneumatic injection, and double L injection), which were applied to various analytes in advanced studies. In addition, Tsai et al. [33-35] presented a microfluidic CE device incorporating a conventional cross-form injection system and an expansion chamber located at the inlet of the separation channel. The geometry of the expansion channel was optimized numerically and the performance of the device was then verified experimentally by carrying out the separation of a mixed Hae III-digested $\varphi x-174$ DNA sample.

Despite the notable contributions of the various injection schemes discussed above, research still continues into the development of enhanced sample injection techniques for CE microchips [36-45]. In a recent study, Blas et al. [37] presented a comparative study of two electrokinetic injection modes in chips: the floating, which has been mainly used up to now, and the dynamic. It appeared that the dynamic injection mode is much more appropriate than the floating mode as it is faster, more reproducible, and leads to more efficient separation for the same injected amount, whatever the amount, because the sample plug is less dispersed. Lee et al. [44] presented a numerical study of the 3-D characteristics of electroosmotic injection and migration of sample species in CE microchip. A non-rectangular cross section resulted in a vertically non-uniform sample plug during the loading stage. Conventional 2-D simulation approach can result in up to $40 \%$ errors in the calculation of injection qualities. It was also found that the 2-D simulation using a proper channel width has a good agreement with the 3-D simulation data.

Figure 1. Schematic illustration of proposed microfluidic chip.

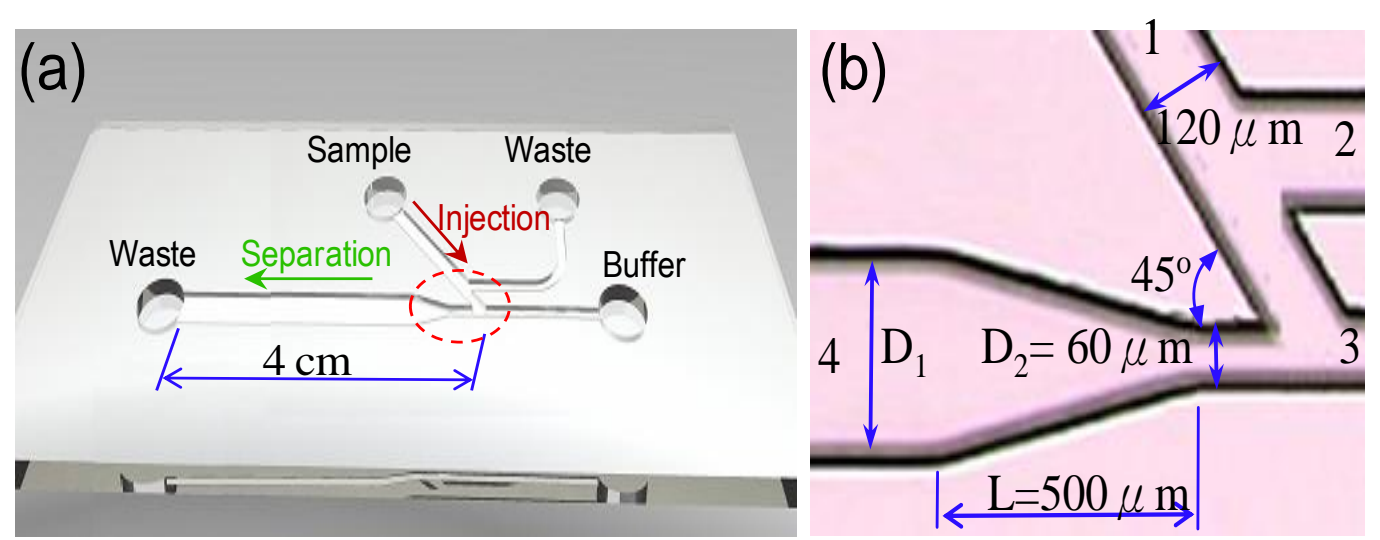

To achieve high-performance detection in a CE microfluidic device, the sample leakage effect and shape of the sample plugs separated into the separation channel is of great importance. Therefore, this study presents an integrated CE microfluidic device that combines a $45^{\circ} \mathrm{U}$-shaped injection system with an expansion chamber at the inlet of the separation channel to eliminate the sample leakage effect and deliver high-quality sample bands into the separation channel. A schematic illustration and photograph of the current CE microfluidic device are presented in Figure 1. This study shows that the 
proposed low leakage injector improves the sample separation and the current expansion chamber advances the high-quality sample bands into the separation channel to enhance the detection performance.

\section{Chip Fabrication and Experimental Setup}

The current microfluidic devices were fabricated on microscope glass slides $\left(76 \times 26 \times 1 \mathrm{~mm}^{3}\right.$, Marienfeld, Germany) using standard photolithographic procedures followed by wet chemical etching. Prior to fabrication, the slides were annealed at $400{ }^{\circ} \mathrm{C}$ for $4 \mathrm{~h}$ to release any internal residual stresses. Initially, microscope slides were cleaned using boiling piranha solution and then patterned with positive photoresist (AZ 4620, Clariant Corp., USA) and UV exposure using a standard photolithography procedure. The patterned glass substrates were then directly immersed in a dilute BOE (6:1), stirred $\mathrm{HF} / \mathrm{NH}_{4} \mathrm{~F}$ bath for 30 min with ultrasonic agitation to generate the $25 \mu \mathrm{m}$ deep microchannels. The fluid-via-holes were drilled by a diamond drill-bit on the etched upper plate. A fusion bonding process was then used to achieve high strength bonding results with a high yield. Briefly, this procedure consisted of the following steps: the two glass flats were carefully aligned and made to cling to each other using deionized (DI) water, the two glasses were held together by atmospheric pressure, and the microchip was sintered above $580{ }^{\circ} \mathrm{C}$ for 20 min with a ramp rate of $5{ }^{\circ} \mathrm{C} / \mathrm{min}^{-1}$. A sealed microfluidic device was able to be formed after bonding the two glass plates. Note that the current authors presented a detailed description of the chip fabrication method for wet chemical etching in a previous study [46,47].

The microfluidic chip performance was monitored by laser-induced fluorescence (LIF). The fluid sample manipulations performed in the current study were observed by mercury lamp induced fluorescence using a charge-coupled device (CCD; model DXC-190, SONY, Japan) for imaging purposes. The study used $10^{-3} \mathrm{M}$ sodium borate ( $\mathrm{pH} 8.5$ Aldrich) as the buffer fluid, and $10^{-4} \mathrm{M}$ Rhodamine B fluorescent dye as the sample. The DNA concentration and electrophoretic separation performance investigations were conducted using a Hae III digested $\varphi x-174$ DNA (Amersham Bioscience, Sweden) sample consisting of eleven fragments (72, 118, 194, 224, 271, 281, 310, 603, 872, 1078 and 1353 base pairs). Detection of the Hae III digested $\varphi x-174$ DNA sample was performed using a CE buffer of 1.2\% HPMC (Hydroxypropyl Methyl Cellulose) in TBE (tris-borate-EDTA) with 1\% SYBR green I (Molecular probes, USA) fluorescent dye. The experimental images were first captured by an optical microscope (mode E400 Nikon, Japan), then filtered spectrally (550 nm cut-on), and finally measured by a CCD device. The voltage switching apparatus was computer-controlled using code written in-house in Labview. Finally, an APD module (Avalanche Photo-Diode, C5640-01, Hamamatsu, Japan) was used to detect the emitted optical signals.

\section{Numerical Modeling}

Numerical simulation of this problem requires solving for the electric potential, zeta potential, ionic concentration, velocity component, and sample concentration throughout the computational domain. A new mathematical model of the electrokinetic transport phenomena in a microfluidic dispenser is developed here with consideration of the spatial gradient of conductivity. The model considers the processes as two-dimensional, which is very common in the modeling of similar processes. Regarding the numerical simulation of electroosmotic flows, the authors previously developed physical models 
based on (a) the Poisson equation $(\psi)$ for the electric potential and zeta potential at the fluid-solid boundary, (b) the Nernst-Planck equations $\left(\mathrm{n}^{+}, \mathrm{n}^{-}\right)$for the positive and negative ionic concentrations, (c) the full Navier-Stokes equations $(\mathrm{u}, \mathrm{v})$ modified to include the effects of the body force due to the electric and charge density, and (d) a sample concentration equation for the sample plug distribution. Note that the detailed expressions of the governing equation, the initial conditions, and the boundary conditions were presented in previous studies by the authors [48].

\section{Results and Discussion}

In a CE microfluidic device, the shape of the delivered sample plug depends primarily on the electroosmotic flow pattern at the intersection of the channels, and plays an important role in determining the resolution of the electrophoresis analysis. Therefore, the injector and separation chamber is one of the key elements in the handling of the sample and delivering process of the CE micofluidic device.

Figure 2 presents the electric potential contours during the injection and separation steps of the $45^{\circ}$ U-shaped injection method in the proposed microfluidic device. The figure shows that the injection and separation steps were performed using an electric field intensity of $300 \mathrm{~V} / \mathrm{cm}$. During the injection step, the sample was loaded from channel 1 to channel 2 by applying an electric field of $300 \mathrm{~V} / \mathrm{cm}$ (channel 1 is kept at a potential $\Phi_{1}$, channel 2 is held at ground potential). Channels 3 and 4 are electrically floating, resulting in a potential below that of $\Phi_{1}$ and, therefore, sample flow from channel 1 towards the intersection 3/4. Therefore, potential gradients existed in channels $1 \rightarrow 3$ and $1 \rightarrow 4$, and this is called the inertial force $(\rho(u . \nabla) u$, where $\rho$ is the density of the sample, $u$ is velocity of sample, and $\nabla$ is operator) (Figure $2 \mathrm{a}$ ). When the sample had completely filled the intersection of channels 3 and 4 , the electrical potential switched immediately to the separation step in which channels 1 and 2 were floating (i.e., $\partial \phi / \partial x=0$ ) and an electric field intensity of $300 \mathrm{~V} / \mathrm{cm}$ was applied across channels $3 \rightarrow 4$. The sample plug was delivered to separation channel 4 , and the sample flow in channel 1 was pulled back by the inertial force from channel 3. Therefore, the sample leakage can be completely eliminated by this proposed $45^{\circ} \mathrm{U}$-shaped injector.

Figure 2. Electrical potential contours in $45^{\circ} \mathrm{U}$-shaped injection method: (a) injection and (b) separation steps with an electric flied of $300 \mathrm{~V} / \mathrm{cm}$.
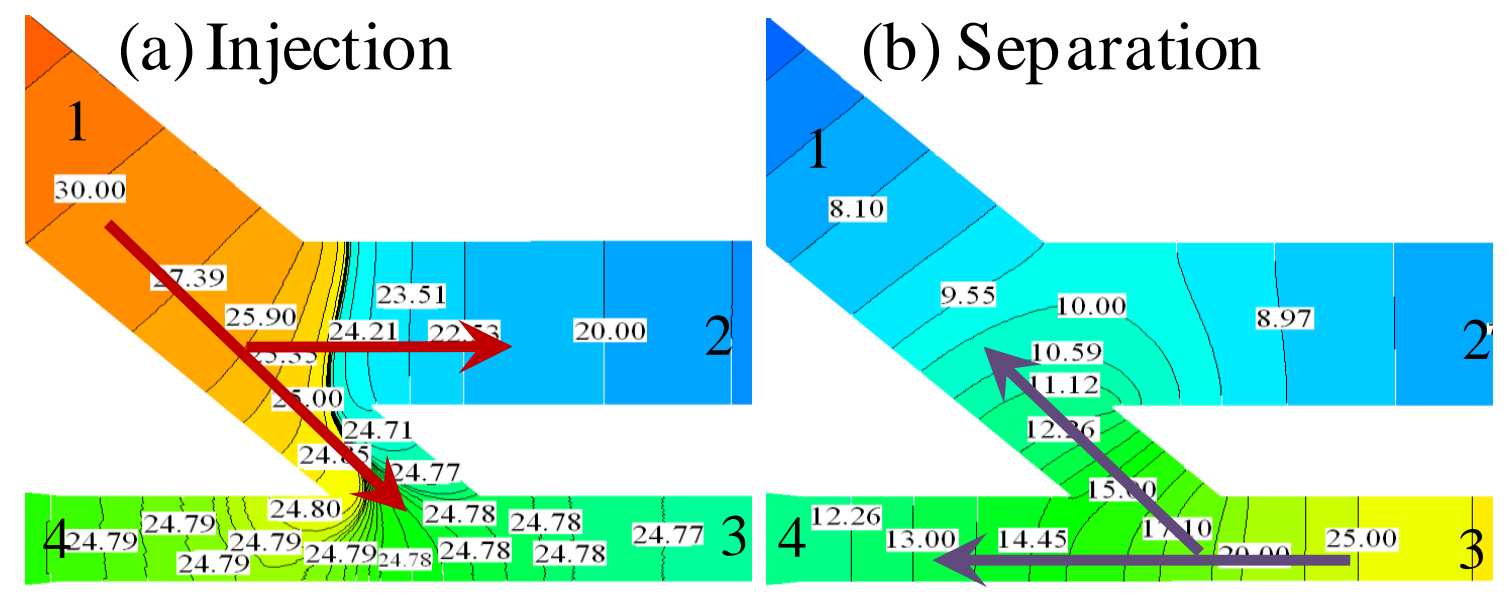
To interpret the effects of sample leakage on the resolution sensitivity of this proposed $45^{\circ}$ U-shaped injector, a constant electric field of $300 \mathrm{~V} / \mathrm{cm}$ was established and a series of injection/separation cycles was performed using a test sample of $10^{-4} \mathrm{M}$ Rhodamine $\mathrm{B}$. The sample was excited within the detection region (located $2.5 \mathrm{~cm}$ downstream from the intersection of channels 3 and 4) using a mercury lamp, and an APD module was then used to detect the resulting optical signals. Figure 3a,b present the acquired optical signals over a series of 8 injection/separation cycles performed with an electric separation field of $300 \mathrm{~V} / \mathrm{cm}$ in cross-shaped and $45^{\circ} \mathrm{U}$-shaped injectors, respectively. For the traditional cross-shaped injector, the baseline begins to amass as the number of cycles increases (Figure 3a). This drift effect is the result of sample leakage from the side channels, clearly reducing the quality of the detection performance. Figure $3 \mathrm{~b}$ presents the optical signals acquired over 8 injection/separation cycles performed using a $45^{\circ} \mathrm{U}$-shaped injector. Compared with the results presented in Figure 3a, it can be seen that the baseline remains stationary over the 8 cycles. This indicates that the injector entirely eliminates sample leakage and is therefore suitable for applications requiring a high detection resolution. Note that more details of the sample leakage effects were presented in previous studies by the authors $[21,23]$.

Figure 3. Acquired optical signals for a series of 8 injection/separation cycles performed with applied electric field of $300 \mathrm{~V} / \mathrm{cm}$ with (a) cross-shaped and (b) $45^{\circ} \mathrm{U}$-shaped injectors, respectively.

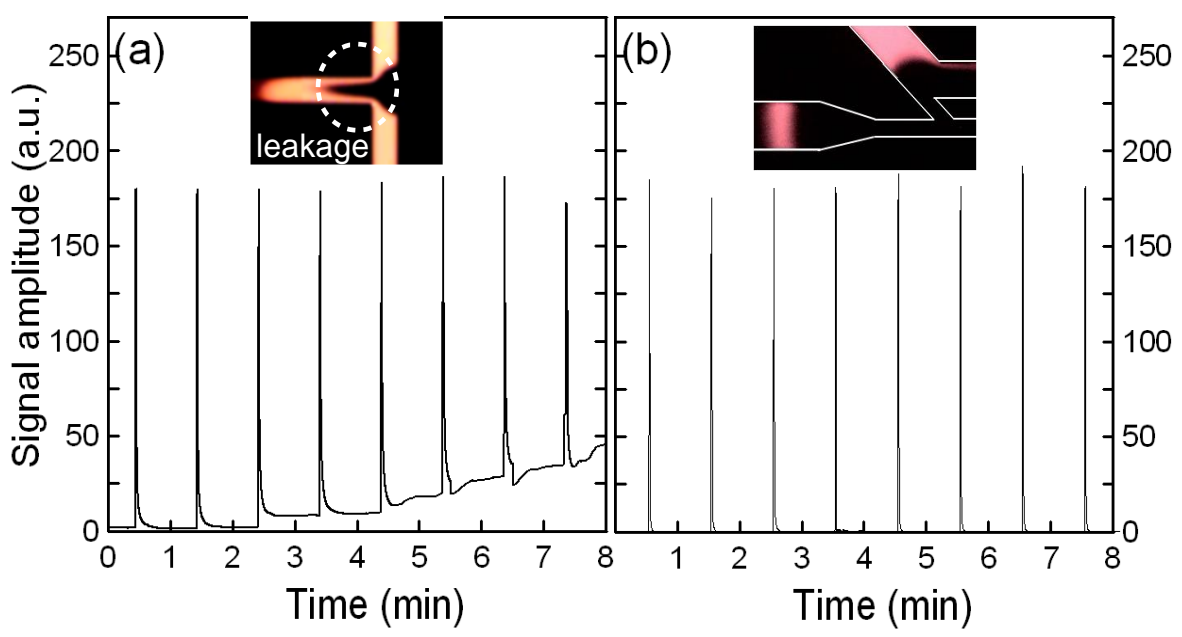

Figure 4 shows the sample plug shape distribution in the separation process and the concentration intensity profiles at the centerline of the sample plug that pass through a constant expansion length (L) of $500 \mu \mathrm{m}$ with different expansion ratios. The expansion length (L) is set to $500 \mu \mathrm{m}$ because this results in a very orthogonal and very small variance sample plug in the separation channel. It is suitable for enhancing the resolution sensitivity of detection. Note that more details on the effect of the expansion length were presented in previous studies by the authors [33-35]. In Figure 4, it is clear that the low expansion ratios of 1 and 2.5 still result in a high intensity sample plug concentration being maintained in the separation channel. However, for an expansion ratio of 1 (i.e., no expansion effect), Figure $4 \mathrm{a}$ indicates that the sample band distribution is rather broad. This reduces the separation efficiency. When the expansion ratio is increased to 2.5 (as shown in Figure 4b), it is clear that the broad sample plug distribution is greatly improved. The concentration intensity remains at its highest 
value, but the sample plug distribution is significantly narrower. When the expansion ratio is increased to 4 (as shown in Figure 4c), the sample plug is dispersed by a larger expansion ratio in the separation channel. The present results indicate that the concentration intensity reduces by about $25 \%$ (compared to Figure $4 \mathrm{a}$ ) at the detection area due to dispersion.

Figure 5 compares the concentration intensity and the relative width of the sample band at a position $1500 \mu \mathrm{m}$ downstream from the intersection of channels 3 and 4 for different expansion ratios. In this figure, the concentration intensity is calculated by averaging the centerline of five sequential sample plugs. A small concentration intensity indicates that the expansion chamber generates too much dispersion of the sample plug, thereby downgrading the detection performance. Considering the relative width of the sample plug, the maximum sample plug width is obtained at an expansion ratio of 1 (see Figure 4a). In general, a narrow sample plug with high concentration intensity results in a higher theoretical plate number and enhanced separation efficiency. From Figure 5, it is clear that an expansion ratio of 2.5 produces the optimal condition between high concentration intensity and a suitable sample plug width.

Figure 4. Sample shape in separation processes and concentration profiles at the centerline of the sample band for a constant expansion length of $500 \mu \mathrm{m}$ and different expansion ratios, i.e., (a) 1 , (b) 2.5 , and (c) 4 .
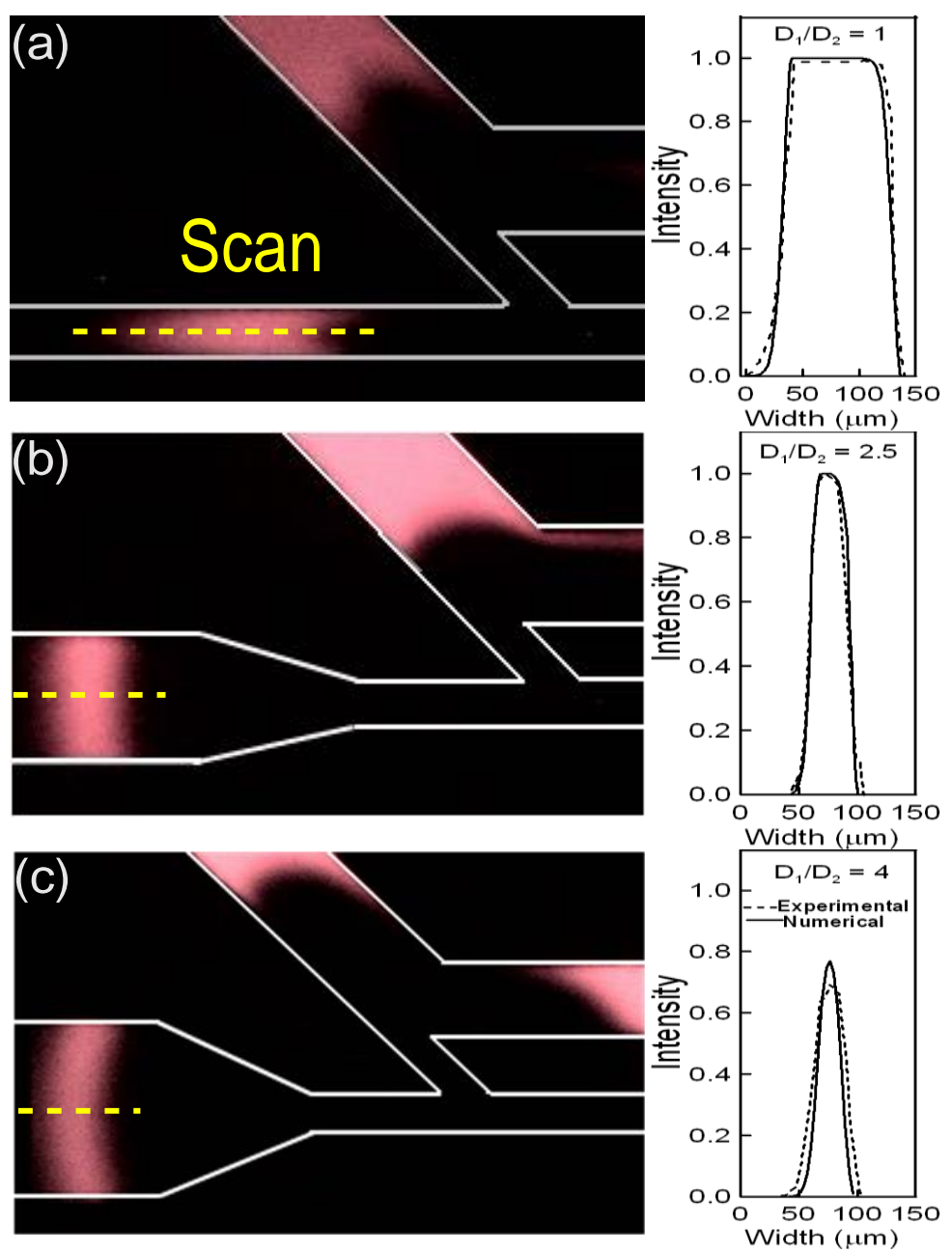
Figure 5. Comparison of concentration intensity and relative width of the sample band for various expanding ratios.

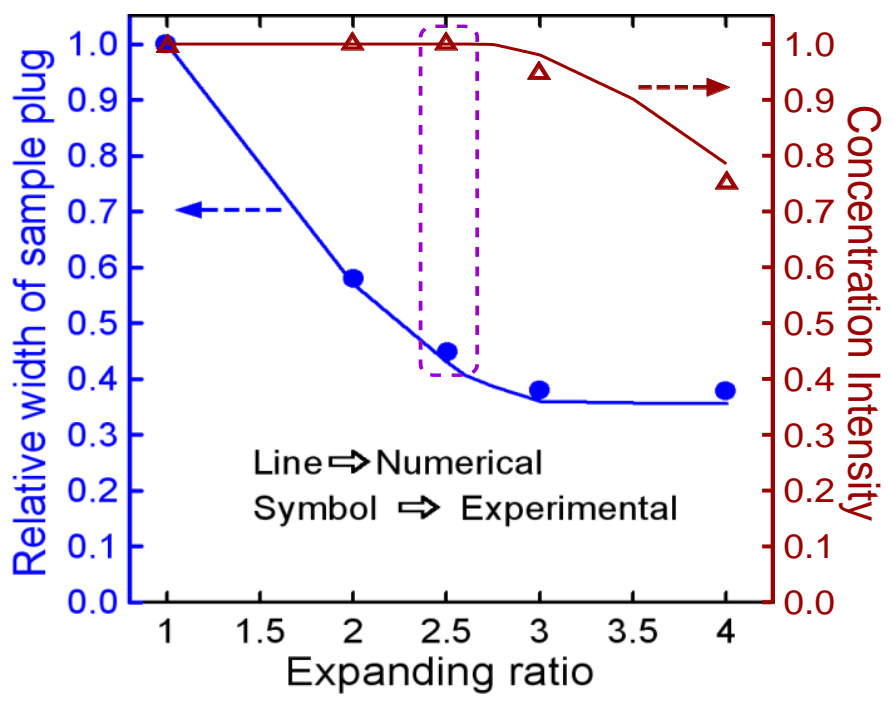

Figure 6a presents electropherograms of five consecutive $\varphi x-174$ DNA separations with a conventional cross-channel injection system utilizing an injection/separation field strength of $300 \mathrm{~V} / \mathrm{cm}$. The DNA sample was a Hae III digested $\varphi \mathrm{x}-174$ DNA consisting of eleven fragments, namely 72, 118, 194, 224, 271, 281, 310, 603, 872, 1078 and 1353 base pairs (bp). In Figure 6a, it can be seen that the baseline begins to drift as the number of injection/separation cycles increases. This phenomenon reflects the effects of sample leakage from injection channels into the separation channel and leads to a significant degradation of the detection resolution. For this sample, eleven distinct peaks were detected in the separation channel. Nevertheless, in the present case, it can be seen that the resolution performance is not desirable, as shown in Figure $6 b$.

Figure 6. Electropherograms of separated $\varphi x-174$ DNA with conventional cross-shaped separation channels (injection and separation field strength set to $300 \mathrm{~V} / \mathrm{cm}$ ).

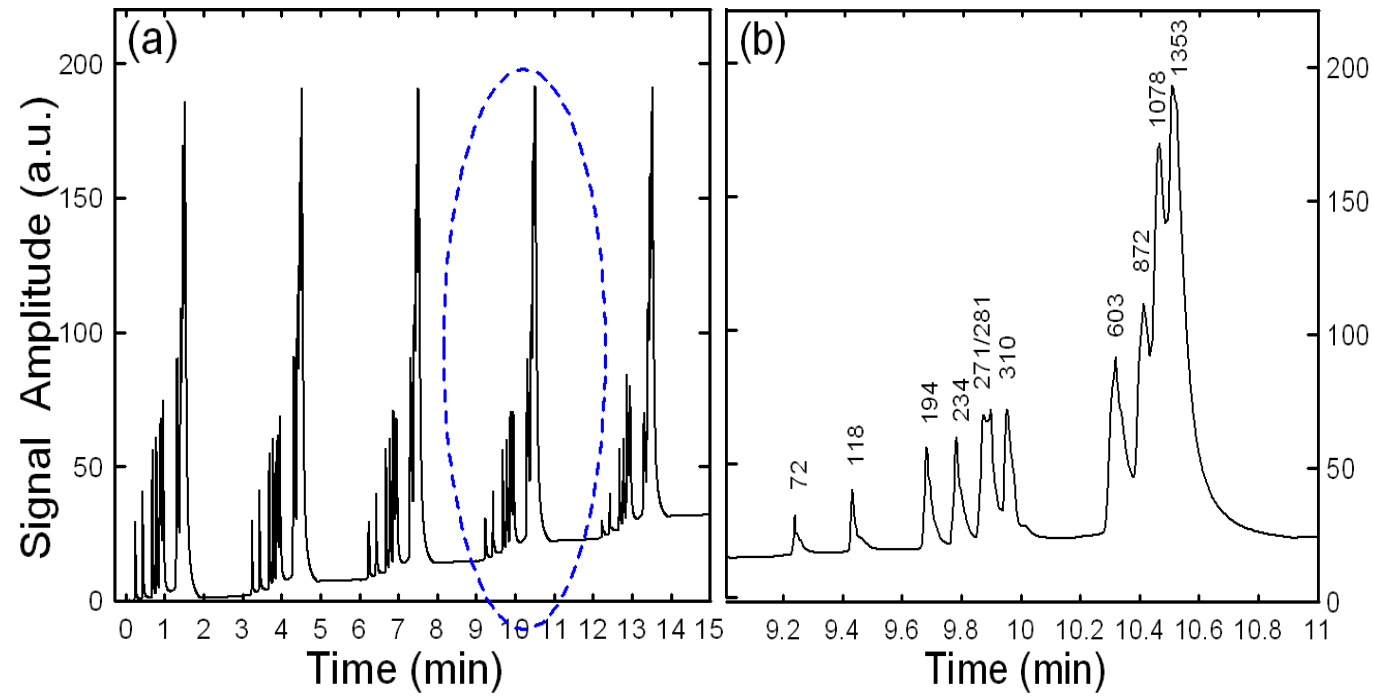

Figure $7 \mathrm{a}$ presents five sequential $\varphi \mathrm{x}-174$ DNA electropherograms when the same sample is separated with this particular $45^{\circ} \mathrm{U}$-shaped injector and expansion chamber (expansion ratio 2.5) with 
an injection/separation voltage of $300 \mathrm{~V} / \mathrm{cm}$. For this sample, the eleven distinct peaks are clearly resolved as shown in Figure $7 \mathrm{~b}$. The results clearly demonstrate that the proposed $45^{\circ}$ injection structure yields improved separation performance.

Figure 7. Electropherogram of separation results for $\varphi x-174$ DNA sample using $45^{\circ}$ U-shaped injector with expansion chamber of expansion ratio 2.5 (injection and separation voltage of $300 \mathrm{~V} / \mathrm{cm})$.

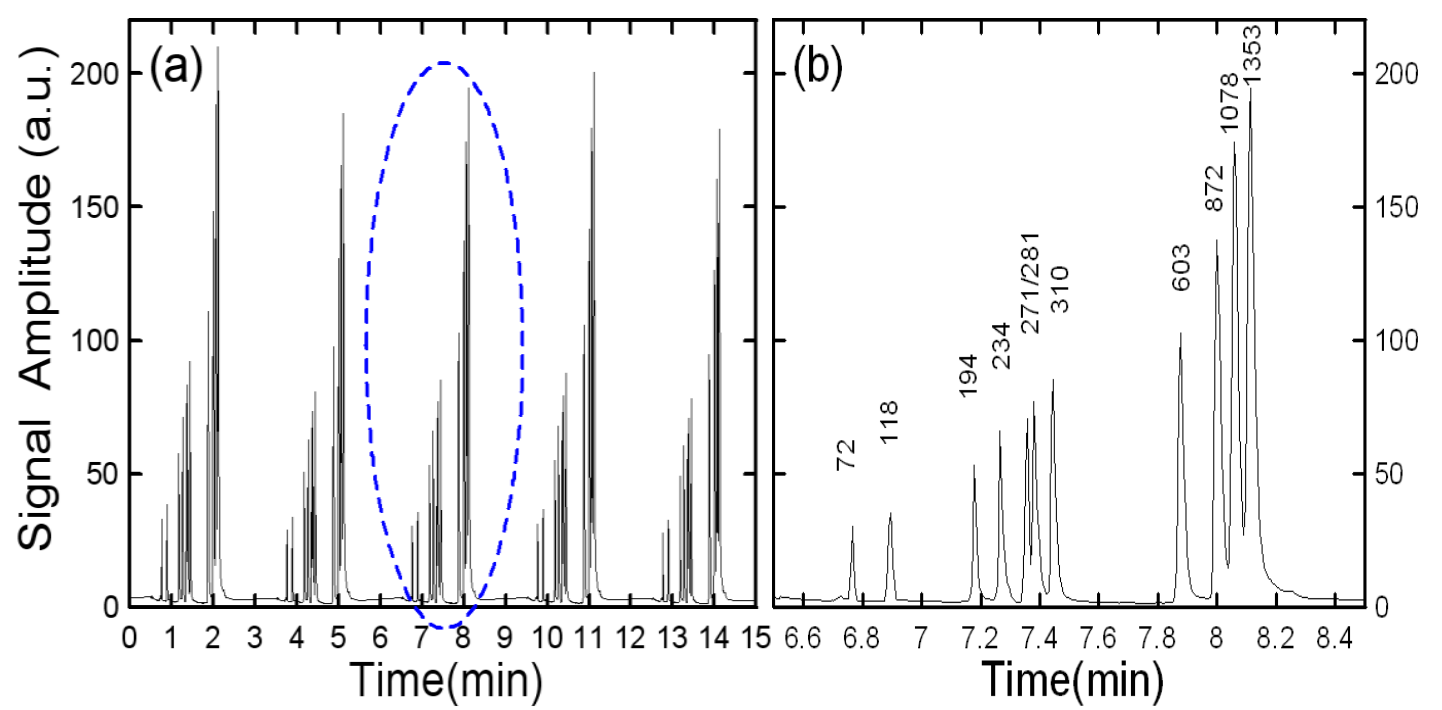

\section{Conclusions}

This study proposes a CE microfluidic device with a novel injection structure with a $45^{\circ} \mathrm{U}$-shaped injector and an expansion chamber at the inlet of the separation channel to improve the sample plug distribution in the separation step and enhance separation efficiency. This study performed the experimental separation of samples consisting of Rhodamine B fluorescent dye and Hae III digested $\varphi x-174$ DNA samples to explore the effects of the $45^{\circ} \mathrm{U}$-shaped injector and expansion chamber on the sample leakage and separation performance. The numerical and experimental results show that the $45^{\circ} \mathrm{U}$-shaped injector can efficaciously avoid sample leakage into the separation channel, and that an expansion chamber with an expansion ratio over than 2.5 results in a higher separation efficiency, as the prolongation of the peaks is reduced. A novel $45^{\circ} \mathrm{U}$-shaped injection system combined with an expansion chamber at the inlet of the separation channel with an expansion ratio of 2.5 was developed and its performance analyzed. The results indicate that this CE microfluidic device significantly prevents sample leakage and transports suitable sample plugs into the separation channel enhancing the detection performance.

\section{Acknowledgements}

The authors would like to thank the financial support provided by the National Science Council in Taiwan. 


\section{References}

1. Fu, L.M.; Lin, C.H. A rapid DNA digestion system. Biomed. Microdevices 2007, 9, 277-286.

2. Tsai, C.H.; Hou, H.H.; Fu, L.M. An optimal three-dimensional focusing technique for micro-flow cytometers. Microfluid. Nanofluid. 2008, 5, 827-836.

3. Liu, D.; Chen, B.; Wang, L.; Zhou, X. On-chip coupling of free-solution transient ITP and CGE for highly efficient separation of dsDNA with variable sample loading amounts. Electrophoresis 2009, 30, 4300-4305.

4. Wen, C.Y.; Lee, T.L.; Tsai, C.H.; Fu, L.M. Rapid magnetic microfluidic mixer utilizing AC electromagnetic field. Electrophoresis 2009, 30, 4179-4186.

5. Wang, J.; Wang, C.; Lin, C.; Lei, H.; Lee, G. An integrated microfluidic system for counting of $\mathrm{CD}^{4+} / \mathrm{CD}^{8+} \mathrm{T}$ lymphocytes. Microfluid. Nanofluid. 2010, 6, 739-749.

6. Xuan, X.; Zhu, J.; Churc, C. Particle focusing in microfluidic devices. Microfluid. Nanofluid. 2010, 9, 1-16.

7. Rosenauer, M.; Vellekoop, M.J. Characterization of a microflow cytometer with an integrated three-dimensional optofluidic lens system. Biomicrofluidics 2010, 4, 043005.

8. Zeng, J. Non-Linear Electrohydrodynamics in Microfluidic Devices. Int. J. Mol. Sci. 2011, 12, 1633-1649.

9. Nieuwstadt, H.; Seda, R.; Li, D.; Fowlkes, J.; Bull, J. Microfluidic particle sorting utilizing inertial lift force. Biomed. Microdevices 2011, 13, 97-105.

10. Guo, W.; Lau, K.; Fung, Y. Microfluidic chip-capillary electrophoresis for two orders extension of adjustable upper working range for profiling of inorganic and organic anions in urine. Electrophoresis 2010, 31, 3044-3052.

11. Cao, B.; Sun, J.; Chen, M.; Guo, Z. Molecular momentum transport at fluid-solid interfaces in MEMS/NEMS: A review. Int. J. Mol. Sci. 2009, 10, 4638-4706.

12. Johns, C.; Breadmore, M.C.; Macka, M.; Ryvolova, M.; Haddad, P.R. Recent significant developments in detection and method development for the determination of inorganic ions by CE. Electrophoresis 2009, 30, S53-S67.

13. Fernandes, P. Miniaturization in Biocatalysis. Int. J. Mol. Sci. 2010, 11, 858-879.

14. Hong, T.F.; Ju, W.J.; Wu, M.; Tai, C.H.; Tsai, C.H.; Fu, L.M. Rapid prototyping of PMMA microfluidic chips utilizing a $\mathrm{CO}_{2}$ laser. Microfluid. Nanofluid. 2010, 9, 1125-1133.

15. Sun, H.; Nie, Z.; Fung, Y.S. Determination of free bilirubin and its binding capacity by HSA using a microfluidic chip-capillary electrophoresis device with a multi-segment circular-ferrofluid-driven micromixing injection. Electrophoresis 2010, 31, 3061-3069.

16. Chuang, H.; Jacobson, S.; Wereley, S. A diffusion-based cyclic particle extractor. Microfluid. Nanofluid. 2010, 9, 743-753.

17. Wang, A.; Cheng, C.; Lin, I.; Lu, F.; Tsai, H.; Lin, C.; Yang, C.; Pan, P.; Kuan, C.; Chen, Y.; Lin, Y.; Chang, C.; Wu, Y.; Kurniawan, T.; Lin, C.; Wo, A.; Chen, L. A novel DNA selection and direct extraction process and its application in DNA recombination. Electrophoresis 2011, 32, 423-430.

18. Oh, K.; Smith, B.; Devasia, S.; Riley, J.; Chung, J. Characterization of mixing performance for bio-mimetic silicone cilia. Microfluid. Nanofluid. 2010, 9, 645-655. 
19. Tofteberg, T.; Skolimowski, M.; Andreassen, E.; Geschke, O. A novel passive micromixer: Lamination in a planar channel system. Microfluid. Nanofluid. 2010, 8, 209-215.

20. Mao, X.; Juluri, B.; Lapsley, M.; Stratton, Z.; Huang, T. Milliseconds microfluidic chaotic bubble mixer. Microfluid. Nanofluid. 2010, 8, 139-144.

21. Hao, G.H.; Duits, M.; Mugele, F. Droplets formation and merging in two-phase flow microfluidics. Int. J. Mol. Sci. 2011, 12, 2572-2597.

22. Fu, L.M.; Lin, C.H. Numerical analysis and experimental estimation of a low leakage injection technique for capillary electrophoresis. Anal. Chem. 2003, 75, 5790-5796.

23. Lin, C.H.; Yang, R.J.; Tai, C.H.; Lee, C.Y.; Fu, L.M. Double-L injection technique for high performance CE detection in microfluidic chips. J. Micromech. Microeng. 2004, 14, 639-646.

24. Tsai, C.H.; Yang, R.J.; Tai, C.H.; Fu, L.M. Numerical simulation of electrokinetic injection techniques in capillary electrophoresis microchips. Electrophoresis 2005, 26, 674-686.

25. Tsai, C.H.; Wang, Y.N.; Lin, C.F.; Yang, R.J.; Fu, L.M. Experimental and numerical analysis of leakage effect for injectors in microfluidic device. Electrophoresis 2006, 27, 4991-4998.

26. Chang, C.L.; Hou, H.H.; Fu, L.M.; Tsai, C.H. A low-leakage sample plug injection scheme for cross-form microfluidic $\mathrm{CE}$ devices incorporating a restricted cross-channel intersection. Electrophoresis 2008, 29, 3135-3144.

27. Zhuang, G.S.; Li, G.; Jin, Q.H.; Zhao, J.L.; Yang, M.S. Numerical analysis of an electrokinetic double-focusing injection technique for microchip CE. Electrophoresis 2006, 27, 5009-5019.

28. Yang, R.J.; Fu, L.M.; Lee, G.B. Variable-volume-injection methods using electrokinetic focusing on microfluidic chips. J. Sep. Sci. 2002, 25, 996-1010.

29. Fu, L.M.; Yang, R.J.; Lee, G.B. Electrokinetic focusing injection methods on microfluidic devices. Anal. Chem. 2003, 75, 1905-1910.

30. Luo, Y.; Wu, D.; Zeng, S.; Gai, H.; Long, Z.; Shen, Z.; Dai, Z.; Qin, J.; Lin, B. Double-cross hydrostatic pressure sample injection for chip CE: Variable sample plug volume and minimum number of electrodes. Anal. Chem. 2006, 78, 6074-6080.

31. Wu, C.H.; Yang, R.J. Improvements on the electrokinetic injection technique for microfluidic chips. Electrophoresis 2006, 27, 4970-4981.

32. Wenclawiak, B.W.; Püschl, R.J. Sample injection for capillary electrophoresis on a micro fabricated device/on chip CE Injection. Anal. Lett. 2006, 39, 3-16.

33. Tsai, C.H.; Hung, M.F.; Chen, L.W.; Chang, C.L.; Fu, L.M. Optimal configuration of CE microchip with expansion chamber in separation channel. J. Chromatogr. A 2006, 1121, 120-128.

34. Fu, L.M.; Leong, J.C.; Lin, C.F.; Tai, C.H.; Tsai, C.H. High performance microfluidic CE devices. Biomed. Microdevices 2007, 9, 405-412.

35. Leong, J.C.; Tsai, C.H.; Fu, L.M. Design of high-resolution analysis technique for capillary electrophoresis microchip. Jap. J. Appl. Phys. 2007, 46, 6865-6870.

36. Hupert, M.L.; Guy, W.J.; Llopis, S.D.; Shadpour, H.; Rani, S.; Nikitopoulos, D.E.; Soper, S.A. Evaluation of micromilled metal mold masters for the replication of microchip electrophoresis devices. Microfluid. Nanofluid. 2007, 3, 1-11.

37. Blas, M.; Delaunay, N.; Rocca, J.L. Comparative study of floating and dynamic injection modes in electrokinetic separative Microsystems. Electrophoresis 2007, 28, 4629-4637. 
38. Blas, M.; Delaunay, N.; Rocca, J.L. Electrokinetic-based injection modes for separative microsystems. Electrophoresis 2008, 29, 20-32.

39. Barz, D.P.J. Comprehensive model of electrokinetic flow and migration in microchannels with conductivity gradients. Microfluid. Nanofluid. 2009, 7, 249-265.

40. Li, B.; Jiang, L.; Xie, H.; Gao, Y.; Qin, J.; Lin, B.C. Development of micropump-actuated negative pressure pinched injection for parallel electrophoresis on array microfluidic chip. Electrophoresis 2009, 30, 3053-3057.

41. Bowen, A.L.; Martin, R.S. Integration of on-chip peristaltic pumps and injection valves with microchip electrophoresis and electrochemical detection. Electrophoresis 2010, 31, 2534-2540.

42. Wu, Z.; Cao, X.; Chen, L.; Zhang, J.; Xia, X.; Fang, Q.; Chen, H. Study on the influence of cross-sectional area and zeta potential on separation for hybrid-chip-based capillary electrophoresis using 3-D simulations. Electrophoresis 2010, 31, 3665-3674.

43. Dossi, N.; Toniolo, R.; Susmel, S.; Pizzariello, A.; Bontempelli, G. A simple approach to the hydrodynamic injection in microchip electrophoresis with electrochemical detection. Electrophoresis 2010, 31, 2541-2547.

44. Lee, D.H.; Farouk, B.; Noh, H.S. 3-D simulation of electroosmotic injection and migration in microchannels: effects of non-rectangular cross section. Sep. Sci. Tech. 2011, 46, 195-204.

45. Yang, M.H.; Wang, S.C.; Cheng, J.C. The optimal control of geometry and voltage parameters on electrokinetic transport to avoid sample leakage. Chromatographia 2011, 73, 567-577.

46. Hou, H.H.; Tsai, C.H.; Fu, L.M.; Yang, R.J. Experimental and numerical investigation into micro-flow cytometer with 3-D hydrodynamic focusing effect and microweir structure. Electrophoresis 2009, 30, 2507-2515.

47. Lin, C.; Lee, C.; Tsai, C.; Fu, L. Novel continuous particle sorting in microfluidic chip utilizing cascaded squeeze effect. Microfluid. Nanofluid. 2009, 7, 499-508.

48. Fu, L.M.; Wang, J.H.; Luo, W.B.; Lin, C.H. Experimental and numerical investigation into the joule heating effect for electrokinetically driven microfluidic chips utilizing total internal reflection fluorescence microscopy. Microfluid. Nanofluid. 2009, 6, 499-507.

(C) 2011 by the authors; licensee MDPI, Basel, Switzerland. This article is an open access article distributed under the terms and conditions of the Creative Commons Attribution license (http://creativecommons.org/licenses/by/3.0/). 